\title{
Proposal of a Permanent Magnet Hybrid-type Axial Magnetically Levitated Motor
}

\author{
Nobuyuki Kurita ${ }^{* a)}$ Member, \\ Hiromu Takada* Non-member, Genri Suzuki* Non-member
}

(Manuscript received July 30, 2014, revised March 11, 2015)

\begin{abstract}
A permanent magnet hybrid-type axial magnetically levitated motor is proposed in this paper. The motor consists of a spherical permanent magnet and an axial type bearingless motor with a tilt control function. The rotor has two permanent magnets on one side, and the motor stator has eight poles, which include eight concentrated windings. The operating principle was investigated through numerical analysis. It was verified that the proposed motor can control the translational motion, inclinational motion, and rotational motion independently. A test rig was fabricated to investigate the control performance. The time responses of each controlled axes were quick. In addition, stable levitated rotation was observed in each actively controlled axes up to $2000 \mathrm{~min}^{-1}$.
\end{abstract}

Keywords: axial flux motor, bearingless motor, magnetically levitation technology, passive magnetic bearing

\section{Introduction}

A magnetically levitated motor (maglev motor) can support and rotate a rotor by using magnetic force with no mechanical contact and, therefore, has many advantages over conventional mechanical bearings ${ }^{(1)(2)}$. In order to accomplish complete magnetic levitation of the rotor, however, it is necessary to control five axes actively. Two radial magnetic bearings and one axial magnetic bearing are usually required, which gives rise to major issues, such as a complicated levitation control system and associated electronics that result in enlarged equipment size.

In order to down size a maglev motor and to simplify the control system, several types of bearing-less motors $(\mathrm{B} \ell \mathrm{M})$ have been proposed and researched, such as a radial- $\mathrm{B} \ell \mathrm{M}^{(3)(4)}$ and an axial- $\mathrm{B} \ell \mathrm{M}^{(5)(6)}$. To increase passive stability, the rotor shape should be a disk shape for radial- $\mathrm{B} \ell \mathrm{M}$ or a long cylindrical shape for axial-B $\ell \mathrm{M}$. Consequently, permanent magnet (PM) size and stator surface area will be decreased due to these rotor shapes. Additionally, these geometries are associated with low rotational torque and weak suspension force.

To solve this problem and increase PM size and stator surface area, several types of axial maglev motors that have inclination motion control function were researched. Shimbo et al. ${ }^{(7)}$ and Chongk-wanyuen el al. ${ }^{(8)}$ used an axial-B $\ell \mathrm{M}$ that has a PM synchronous motor on the upper side and a reluctance motor on the lower side. Osa et al. ${ }^{(9)}$ and Takada et al. ${ }^{(10)}$ used an identical stator on both sides of the rotor. Since these axial-B $\ell \mathrm{Ms}$ required two stators to control the tilt motion, the number of amplifiers required for levitation control increases, and the levitation control becomes more complex.

a) Correspondence to: Nobuyuki Kurita. E-mail: nkurita@gumau.ac.jp

* Gunma University

1-5-1, Tenjin-cho, Kiryu, Gunma 376-8515, Japan

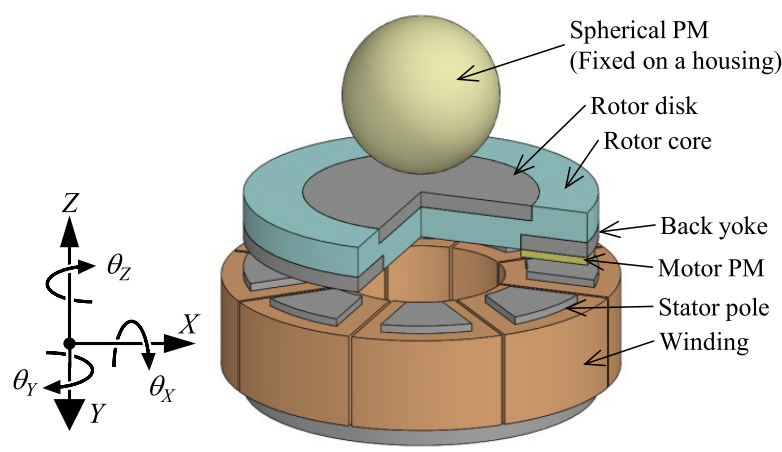

Fig. 1. Schematic of proposed maglev motor

As an alternative, the research proposed in this paper details a PM hybrid type axial maglev motor, shown schematically in Fig. 1. The maglev motor consists of a spherical permanent magnet as a upper side stator and an axial-B $\ell \mathrm{M}$ as a lower side stator, and a rotor. The spherical PM is fixed on housing in order to produce upward attractive force on the rotor. The axial-B $\ell \mathrm{M}$ controls rotation, axial translation and tilt motion actively. A spherical permanent magnet was used in order to produce axial directional upward attractive force (z) to levitate a rotor with zero-bias current. It is also used to minimize the negative torque produced to tilt direction $\left(\theta_{x}\right.$, $\theta_{y}$ ). A rotor disk diameter is bigger than the spherical permanent magnet diameter, so the attractive force of the permanent magnet won't limit movement of the rotor in radial direction $(x, y)$. Moreover, magnetic pole of the spherical permanent magnet doesn't change when the rotor is rotating, so permanent magnet won't produce negative rotating torque in rotational direction $\left(\theta_{z}\right)$. The number of amplifiers required is only eight. This is half as many as compared with the double stator type magnetically levitated axial gap motor ${ }^{(10)}$. Moreover, the structure of the proposed motor is very simple. The 
motor is proposed to develop a left ventricular assist device or a small fan in particular.

The operating principle is described below, as is an FEM magnetic analysis that indicates the proposed maglev motor can control each degree of freedom independently. Based on this theoretical framework, an experimental setup was fabricated. Magnetic levitation was achieved and the control performance of the maglev motor was also investigated.

\section{Operating Principle}

The proposed maglev motor consists of a spherical PM and a conventional axial-B $\ell \mathrm{M}$. The rotor of the axial- $\mathrm{B} \ell \mathrm{M}$ has two crescent shape PMs, and the stator has eight salient poles with a concentrated winding on each pole. The spherical PM as the upper side stator always produces upward attractive force, while the motor PMs always produce downward attractive force. Rotation, axial translation, tilt motion are controlled actively by the interaction between the magnetic field produced by the motor PM and the magnetic field produced by the control currents. Figure 2 is top view of the maglev motor. The spherical PM, the rotor disk, the rotor core, the back yoke and windings were omitted. Motor PM was depicted translucently. Characters $N_{P M}$ and $S_{P M}$ written on the motor PMs means the magnetic pole at the lower side airgap. The flux density distribution produced by the motor PMs in lower-side airgap is described by Eq. (1).

$$
B_{p m}(\theta, t)=B_{P M} \cos (\omega t+\theta)
$$

where $B_{P M}(\mathrm{~T})$ is the maximum value of the rotor PM flux density, $\omega(\mathrm{rad} / \mathrm{s})$ is rotation speed, $t(\mathrm{sec})$ is time, and $\theta(\mathrm{rad})$ is angle. To control the rotor rotation $\theta_{z}$, a two-pole rotating magnetic field of phase difference $\psi(\mathrm{rad})$ is generated by the stator windings.

The flux density $B_{\theta z}$ of the rotational control current can be written as (2)

$$
B_{\theta z}(\theta, t)=-B_{\Theta Z} \cos (\omega t+\theta+\psi)
$$

where $B_{\Theta Z}$ is the maximum value of the flux density produced by the motor current.

A schematic of magnetic pole arrangements is shown in Fig. 3(b). A two-pole rotating magnetic field of phase difference $\psi$ (rad) results in a rotating torque in a counterclockwise direction $\theta_{z}$.

To control the axial translational motion $z$, the stator windings should produce a two-pole magnetic field which has the same phase as the PM magnetic field. The flux density $B_{z}$ created by the axial direction control current would thus described as (3)

$$
B_{z}(\theta, t)=-B_{Z} \cos (\omega t+\theta)
$$

where, $B_{Z}$ is the maximum value of the flux density produced by the axial direction control current. Figure 3(b) shows the magnetic pole arrangements of the rotor PM and the axial direction control current. Here, the left side poles are magnetized to $N$ poles at the same time the right side poles are magnetized to $S$ poles, so an attractive force acts between the rotor and the stator, resulting in a downward suspension force $F_{Z}$ acting on the rotor. Meanwhile, to control the tilt motion $\theta_{x}$ and $\theta_{y}$, a four-pole magnetic field should be generated by

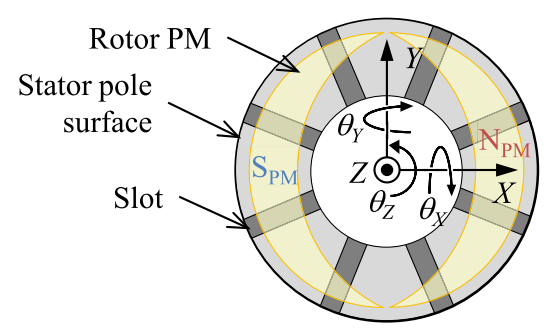

Fig. 2. Top view of the maglev motor and coordinate system

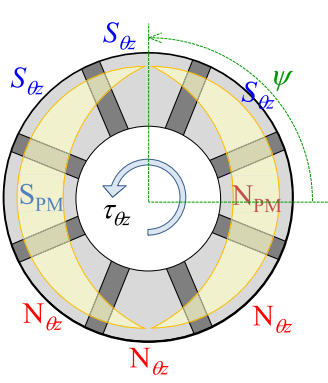

(a) Rotation control

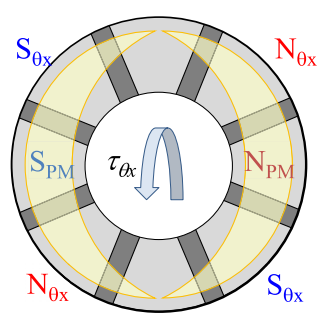

(c) $\theta_{x}$ tilt motion control

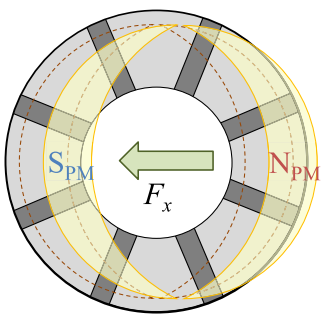

(e) Restoring force $F_{x}$

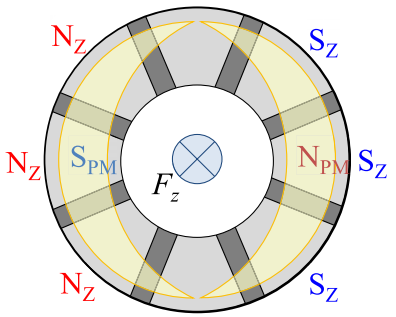

(b) Translation control

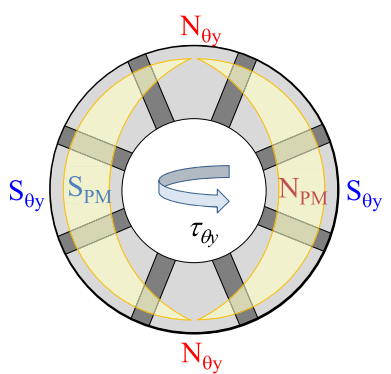

(d) $\theta_{y}$ tilt motion control

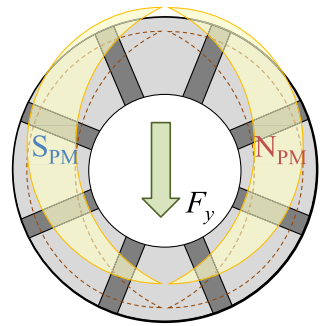

(f) Restoring force $F_{y}$
Fig. 3. Magnetic pole arrangement

the control current. The flux density $B_{\theta x}$ and $B_{\theta y}$ for $\theta \mathrm{x}$ and $\theta \mathrm{y}$ tilt motion control are given by (4)

$$
\begin{aligned}
& B_{\theta x}(\theta, t)=B_{\Theta} \sin (\omega t+2 \theta) \\
& B_{\theta y}(\theta, t)=-B_{\Theta} \cos (\omega t+2 \theta)
\end{aligned}
$$

where $B_{\Theta}$ is the maximum value of the flux density produced by the tilt control current. In Fig. 3(c), the left near side pole and the right far side pole are magnetized to $\mathrm{N}$ poles, and the left far side pole and the right near side pole are magnetized to $\mathrm{S}$ poles. In this configuration, an attractive force acts on the rotor at the near side and a repulsive force acts in far side. As a consequence of these forces, a restoring torque $\tau_{\theta x}$ acts in $\theta_{x}$ direction. A restoring torque $\tau_{\theta y}$ for $\theta_{y}$ direction is produced in the same way. In Fig. 3(d), magnetic pole arrangement for $\tau_{\theta y}$ is depicted. Additionally, radial direction translational motions are stabilized passively. Radial directional restoring force $F_{r x}, F_{r y}$ for displacement $x, y$ are shown in Figs. 3(e), 
Table 1. Principle sizes of the FEM model

Rotor

\begin{tabular}{|l|c|}
\hline Disk diameter & $\phi 30 \mathrm{~mm}$ \\
\hline Disk thickness & $1 \mathrm{~mm}$ \\
\hline Back yoke OD & $\phi 45 \mathrm{~mm}$ \\
\hline Back yoke ID & $\phi 27 \mathrm{~mm}$ \\
\hline Back yoke thickness & $2 \mathrm{~mm}$ \\
\hline PM thickness & $1 \mathrm{~mm}$ \\
\hline Rotor mass & $104.5 \mathrm{~g}$ \\
\hline Inertia about x-axis & $43.6 \mathrm{~kg} \cdot \mathrm{mm}^{2}$ \\
\hline Inertia about y-axis & $44.2 \mathrm{~kg} \cdot \mathrm{mm}^{2}$ \\
\hline Inertia about z-axis & $86.0 \mathrm{~kg} \cdot \mathrm{mm}^{2}$ \\
\hline
\end{tabular}

Motor stator

\begin{tabular}{|c|c|}
\hline OD & $\phi 45 \mathrm{~mm}$ \\
\hline ID & $\phi 27 \mathrm{~mm}$ \\
\hline Pole height & $13 \mathrm{~mm}$ \\
\hline Slot width & $4 \mathrm{~mm}$ \\
\hline Winding number & 129 turns/pole \\
\hline \multicolumn{2}{|l|}{ Spherical PM size } \\
\hline Diameter & $\phi 20 \mathrm{~mm}$ \\
\hline
\end{tabular}

(f). When rotor was displaced in $\mathrm{x}$ or the $\mathrm{y}$-direction, a radius component of the attractive force between the rotor PM and stator core acts on rotor. This radial directional force is used as restoring force.

\section{Magnetic Field Analysis}

3.1 Validation of Control Force Independency In order to verify that rotation control, translation control, and inclination control are not cross-coupled, an FEM magnetic field analysis was carried out; principle sizes of the model are listed in Table 1. The rotor was located at the geometric center between the spherical PM and motor stator. The airgap between the rotor and the stator was $3 \mathrm{~mm}$. By applying each control current (rotation control current $I_{\theta z}$, axial direction translational motion control current $I_{z}$, and tilt control currents $I_{\theta x}, I_{\theta y}$ ) to each coil according to (2), (3) and (4), the rotation torque $\tau_{\theta z}$, translation suspension force $F_{Z}$ and restoring torques $\tau_{\theta x}, \tau_{\theta y}$ were calculated.

When the phase difference of the stator pole and rotor pole is $\psi=\pi / 2$, the relationships between rotational torque $\tau_{\theta z}$ and each control current are as shown in Fig. 4(a). Specifically, a linear relationship exists between the rotational torque $\tau_{\theta z}$ and rotation control current $I_{\theta z}$, but the rotational torque created by translation control current and tilt control current are negligibly small. Figure 4(b) shows the relationships between the axial direction suspension force $F_{Z}$ and each control current. When the upper and lower airgaps are both $3 \mathrm{~mm}$, the resulting attractive force by the motor PMs is stronger than the attractive force of the spherical PM by $1.8 \mathrm{~N}$. The suspension force $F_{Z}$ is approximately proportional to the translational motion control current $I_{z}$. With a low control current of $\pm 1.5 \mathrm{~A}$, the suspension force produced by the rotation and both tilt control currents are negligible. However, when the current of more than $\pm 1.5 \mathrm{~A}$ is applied, the rotation and $\theta_{x}$ tilt control currents produce a suspension force. This force is due to the interaction of the flux produced by the spherical PM on the flux produced by the rotor PM, which results in the flux

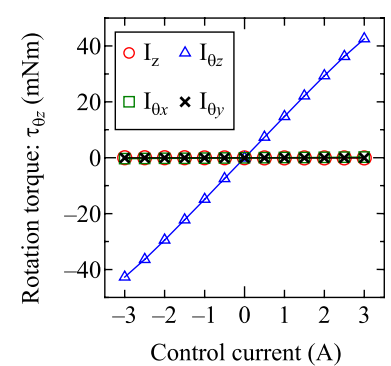

(a) Rotation torque
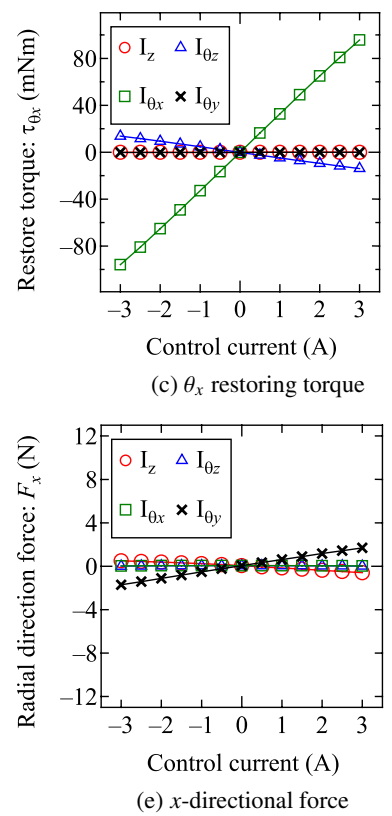

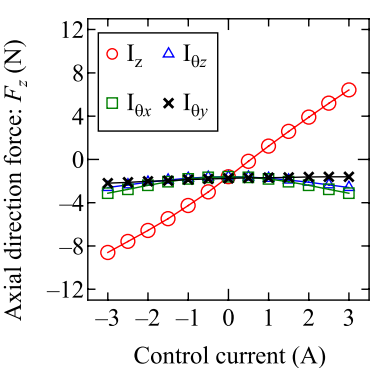

(b) Suspension force
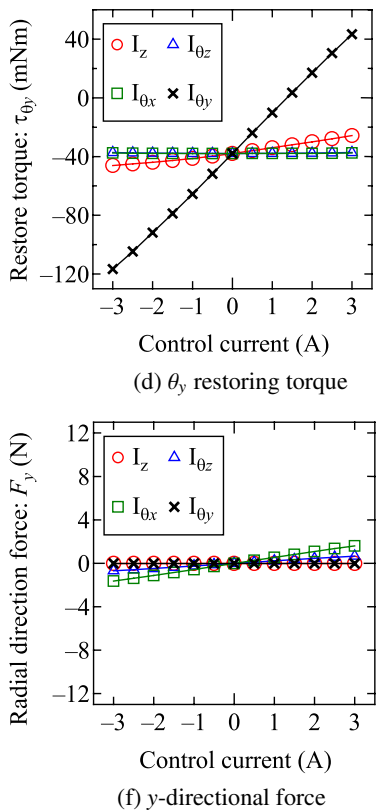

Fig. 4. Analytical result of interference of control current

density distribution in the lower side airgap varying from the ideal sinusoidal wave.

The relationship between restoring torque $\tau_{\theta x}$ and each control current are shown in Fig. 4(c). Restoring torque $\theta_{x}$ is mainly produced by tilt control current $I_{\theta x}$, however, the rotational motion control current $I_{\theta z}$ does contribute some torque. Figure 4(d) shows a similar relationship between restoring torque $\tau_{\theta y}$ and each control current. The variation of the flux density distribution of the motor PM caused by the flux of the spherical PM produced a constant torque of $37 \mathrm{mNm}$ in the $\theta_{y}$ direction. As seen for the other tilt direction, the restoring torque $\theta_{y}$ is mainly produced by tilt control current $I_{\theta y}$, but some contribution also comes from the translation motion control current $I_{z}$. Those interferences of torque and force are again due to the variation of the flux density distribution of the motor PM. We postulate that a closed loop magnetic circuit for the upper side PM would decrease the mutual interference of torque and force.

Finally, Figs. 4(e) and (f) show the relationship between radial directional force $F_{x}, F_{y}$ and each control current. Interferences of torque and force are observed. In this case $x$ directional force produced by translation motion control current $I_{z}$ and $y$-directional force produced by the rotation control current $I_{\theta z}$ are thought to be due to the variation of the flux density distribution of the motor PM. Meanwhile, the $x$ directional force $F_{x}$ produced by tilt control current $I_{\theta y}$ and and $y$-directional force $F_{y}$ produced by tilt control current $I_{\theta z}$ 


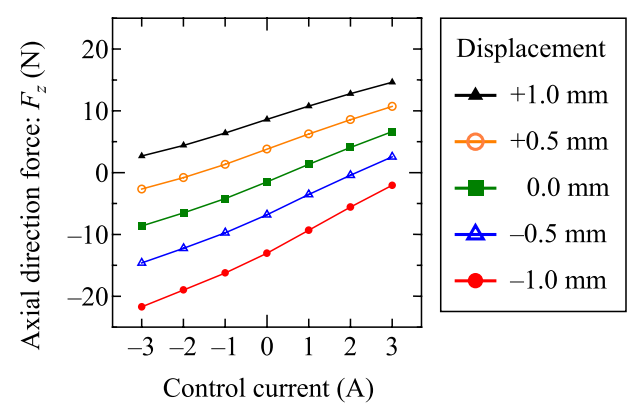

Fig. 5. Axial directional force variation with, displacement and control current

are thought to be due to the structure of maglev motor.

Forces $F_{x}, F_{y}$ in the radial direction were not observed in the double stator type maglev motor ${ }^{(10)}$ because they were canceled due to the symmetric stator design. In a physical setup, perfect symmetry is not achievable, but the magnitude of this force issmall enough such that it does not pose a significant problem for magnetic levitation control; an additional electromagnet could be used to reduce the radial forces if desired.

3.2 Validation of Controllable Range In order to verify the controllable range of the rotor movement, another FEM magnetic field analysis was carried out. Initially, the airgap between the rotor and the stator was $3 \mathrm{~mm}$ and the rotor was located at the geometric center between the spherical PM and motor stator. A suspension force coefficient of $2.59 \mathrm{~N} / \mathrm{A}$ was obtained at the geometric center. The rotor was then moved axially ( $z$-direction) in the range of $\pm 1 \mathrm{~mm}$, and the control current of $\pm 3 \mathrm{~A}$ was applied in each position; analytical results are shown in Fig. 5. According to the analytical result, it can be estimated that the negative $3 \mathrm{~A}$ control current can produce negative directional force even when the rotor is displaced toward the positive direction about $0.74 \mathrm{~mm}$, and the positive $3 \mathrm{~A}$ control current can produce positive directional force when the rotor is displaced toward the negative direction about $-0.78 \mathrm{~mm}$. Thus, the controllable rage of the axial direction can be estimated from $0.74 \mathrm{~mm}$ to $-0.78 \mathrm{~mm}$. It should be noted that the controllable range in the positive and negative direction was not same due to the difference between the magnetic equivalent point and the geometric center.

Next, the rotor was tilted around $x$ and $y$ axes in the range of $\pm 4.5 \mathrm{deg}$, and the control current of $\pm 3 \mathrm{~A}$ was applied in each angle; results are shown in Fig. 6. According to the analytical result of the restoring torque $\theta_{x}$ shown in Fig. 6(a), it can be estimated that $\pm 2.23 \mathrm{~A}$ control current can produce sufficient control torque when the rotor tilted about $4.5 \mathrm{deg}$. Thus, the controllable rage of the tilt motion can be estimated from $\pm 4.5 \mathrm{deg}$. A restoring torque coefficient of $32.2 \mathrm{mNm} / \mathrm{A}$ was obtained at the geometric center. In contrast, the restoring torque coefficient for $\theta_{y}$ was $26.9 \mathrm{mNm} / \mathrm{A}$ at the geometric center. As shown in Fig. 6(b), it can be estimated that a negative $2.74 \mathrm{~A}$ control current can produce negative directional torque when the rotor tilts toward the positive direction about $4.5 \mathrm{deg}$, and positive $3 \mathrm{~A}$ control current can produce positive directional torque when the rotor tilts toward the negative direction about $-1.79 \mathrm{deg}$. Thus, the controllable range of the restoring torque of $\theta_{y}$ can be estimated from $4.5 \mathrm{deg}$ to $-1.79 \mathrm{deg}$. Again, the asymmetry is due to the variation of

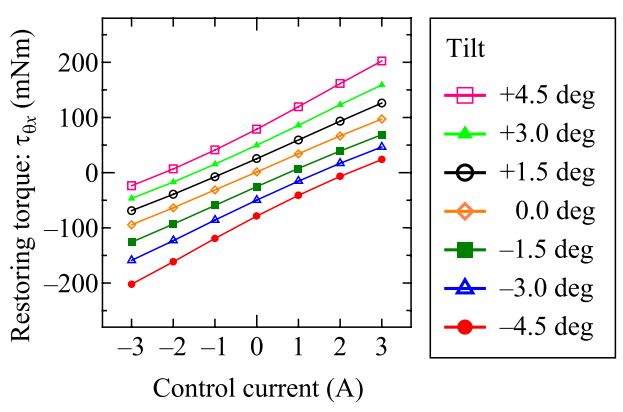

(a) Restoring torque around $x$ axis

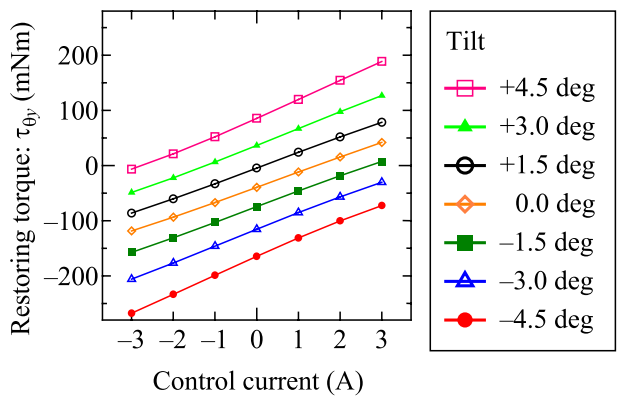

(b) Restoring torque around $y$ axis

Fig. 6. Restoring torque variation with, tilt and control current

the flux density distribution of the motor PM caused by the flux of the spherical PM.

\section{Experimental Results}

4.1 Experimental Setup According to the analytical result, a simple experimental setup was designed and fabricated. Figure 7(a) shows the upper and lower stator. The upper stator consists of spherical PM and PM holder, while the lower stator consists of the eight pole stator and concentrated winding. Figure 7(b) shows top and bottom views of the rotor. The rotor disk is made of ferromagnetic material in order to produce upward attractive force toward the upper stator; the disk surrounded by a sensor target installed around the rotor core. Two crescent shaped PMs were added to the underside produce magnetic fields for rotation and downward attractive force. A Fig. 7(c) shows the entire unit of the fabricated test rig. Although omitted in the figure, three eddy current type gap sensors were evenly spaced along the axial direction in order to detect axial directional displacement and tilt motion of the rotor. A control system of the motor is shown in Fig. 8. The rotor displacement $z_{1}, z_{2}$ and $z_{3}$ detected by three eddy current type gap sensors are used to calculate axial displacement of the center of the gravity $z$ and the rotor tilts $\theta_{x}, \theta_{y}$. Calculated displacement signals are fed to a digital signal processor via $\mathrm{AD}$ converter. Three independent digital PID controllers for these displacement signals are constructed by the DSP. The PID controllers calculate control current reference values for each axis. The PID gains were tuned by trial and error method based on the time responses of the rotor. The sampling time interval was $\mathrm{t}=0.1 \mathrm{msec}$. Translation control gains for levitation control were as follows; proportional gain $K_{Z P}=6.2 \mathrm{~A} / \mathrm{mm}$, derivative gain $K_{Z D}=0.015 \mathrm{~A} \cdot \mathrm{sec} / \mathrm{mm}$, Integrated gain $K_{Z I}=$ $3.0 \mathrm{~A} /(\mathrm{mm} \cdot \mathrm{sec})$. Tilt control gains are follows; proportional gain $K_{\theta P}=5.0 \mathrm{~A} / \mathrm{deg}$, derivative gain $K_{\theta D}=0.011 \mathrm{~A} \cdot \mathrm{sec} / \mathrm{deg}$, 


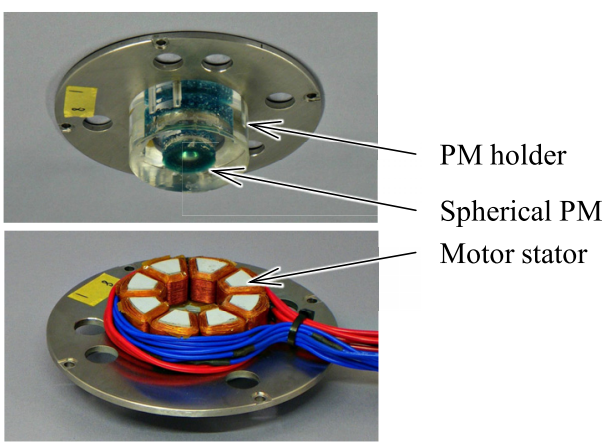

(a) Photo of the upper and lower stator

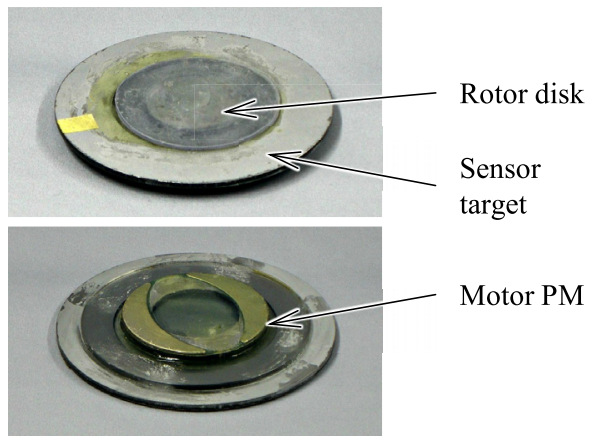

(b) Photo of the rotor

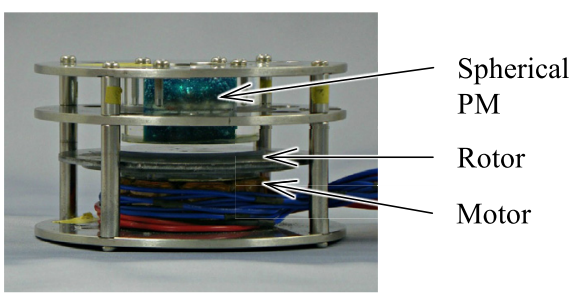

(c) Entire unit

Fig. 7. Photo of the fabricated experimental setup

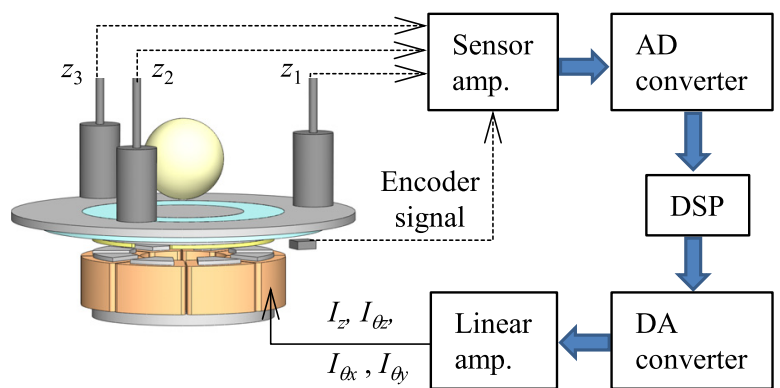

Fig. 8. Diagram of the control system of the motor

Integrated gain $K_{\theta I}=3.0 \mathrm{~A} /(\mathrm{deg} \cdot \mathrm{sec})$. Although omitted in the figure, forty nine small PMs were attached underneath the sensor target. Forty eight PMs are attached evenly spaced and one PM is attached inner side of these PMs in order to works as index. And three hall ICs (i.e. A-Phase, B-Phase and Index) were installed to compose an angular encoder.

4.2 Static Force Characteristics Static force in the axial direction was measured and compared with the analytical results. Figure 9 shows measurement results when the rotor was displaced $\pm 1.0 \mathrm{~mm}$ and a control current of $\pm 2.4 \mathrm{~A}$ was applied. The measured force was weaker than the analytical result by about $20 \%$ to $30 \%$. This is considered due to the error of material characteristics and roughness of the mesh size at the wide air gap of $3 \mathrm{~mm}$. Suspension force co-

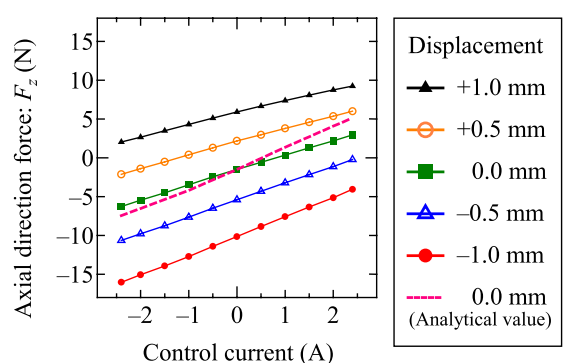

Fig. 9. Measured result of axial directional force, displacement and control current

efficient of $1.92 \mathrm{~N} / \mathrm{A}$ was obtained at the geometric center. This value is $74.1 \%$ of the analytical result.

In accordance with the analytical result, the negative $2.4 \mathrm{~A}$ control current could produce negative directional force even when the rotor was displaced toward the positive direction about $0.74 \mathrm{~mm}$. However, the controllable range to the negative directional movement became especially narrow by comparison with the analytical result. The axial range was estimated to be $0.77 \mathrm{~mm}$ to $-0.44 \mathrm{~mm}$. The restoring torque was not measured but we expect it may be weaker than that predicted by the analytical result, similar to the force results in the axial direction.

4.3 Levitation Control Performance Rotor levitation control with 0 min- 1 rotation was achieved. To control the axial translation motion, the tilt, and the rotation independently, eight sets of linear amplifiers were required. Once the rotor was levitated stably, an impulse disturbance was applied, and the rotor displacement and control current were recorded. Duration of an impulse disturbance signal was fixed on $4 \mathrm{msec}$ and the strength was changed. The rotor was displaced $0.1 \mathrm{~mm}$ in axial direction, $0.5 \mathrm{deg}$ in tilt $\theta_{x}$ direction, $1 \mathrm{~mm}$ in radial direction. As shown in Fig. 10(a), the response of the axial controller is fast; the rotor vibration dropped to less than $\pm 5 \%$ of the maximum displacement within about $0.02 \mathrm{sec}$ in response to an impulse input which moved the rotor about $0.1 \mathrm{~mm}$ in the $\mathrm{z}$-direction. Moreover, since there was almost no rotor vibration in the inclination direction $\left(\theta_{x}, \theta_{y}\right)$ by the z-direction perturbation, the axial direction translation control does not appear to affect the control performance of the tilt control. The response of the tilt controller was measured to be similarly quick for an impulse tilting input of about $0.4 \mathrm{deg}$ in the $\theta_{x}$-direction (Fig. 10(b)); the rotor's rotational vibration became less than $\pm 5 \%$ of the maximum tilt within about $0.03 \mathrm{sec}$. It was also verified that the tilt control also does not affect the control performance of the axial translation control. Figure 10(c) shows the rotor behavior in response to an impulse input which moved the rotor about $1.0 \mathrm{~mm}$ in the radial direction. In this case, the radial vibration took nearly $0.9 \mathrm{sec}$ to fall below $\pm 5 \%$ of the maximum displacement. The slow settling time of the radial direction control is a consequence of the radial direction being stabilized passively by the restoring force produced by the axial attractive force (rather than employing an active stabilization control).

4.4 Levitated Rotation Performance In order to clarify levitated rotation characteristics, vibration amplitude in the axial translation, tilt and radial axes were measured 

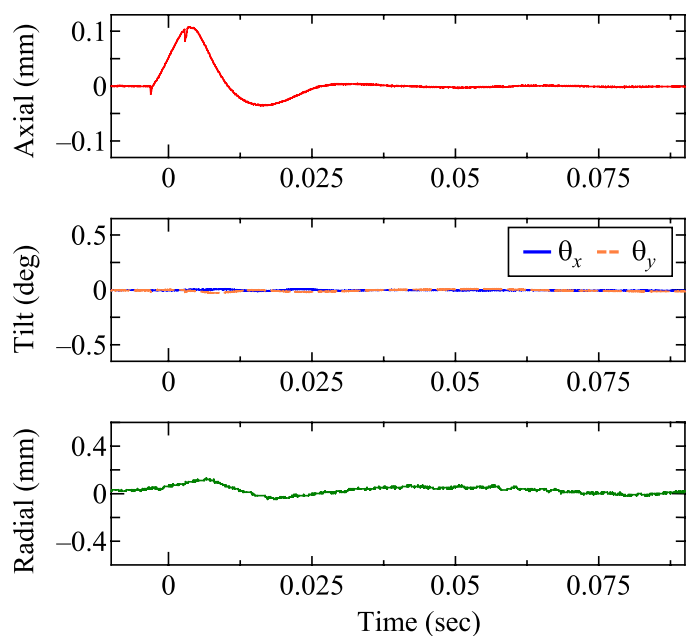

(a) Disturbance input: Axial direction $z$
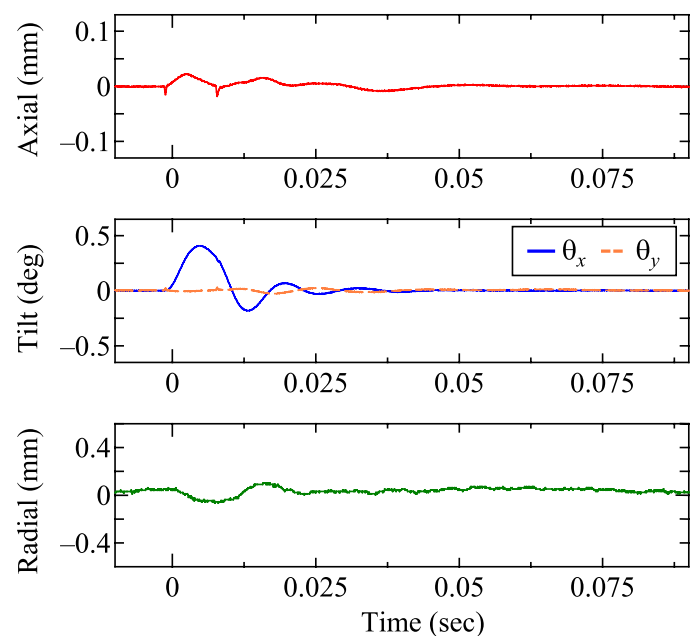

(b) Disturbance input: Tilt $\theta_{x}$
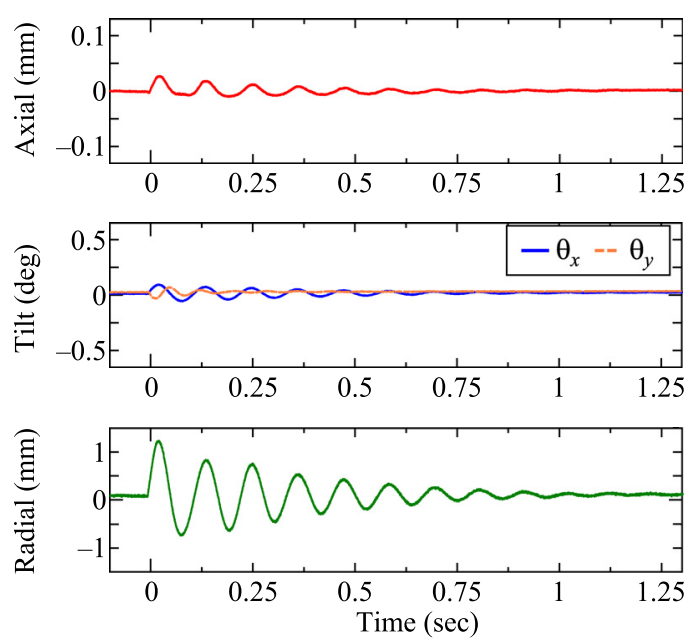

(c) Disturbance input: Radial direction $x$

Fig. 10. Impulse response result

at rotational speeds from $0 \mathrm{~min}^{-1}$ to $2000 \mathrm{~min}^{-1}$. The rotor speed was increased stepwise by $100 \mathrm{~min}^{-1}$ after the rotor reached a steady state speed, and the vibration amplitude of the rotor was recorded (Fig. 11).

The rotor vibration of radial direction was increased at speeds of $600 \mathrm{~min}^{-1}$ to $900 \mathrm{~min}^{-1}$ due to the weak restoring force of the passive control in the radial direction. Although

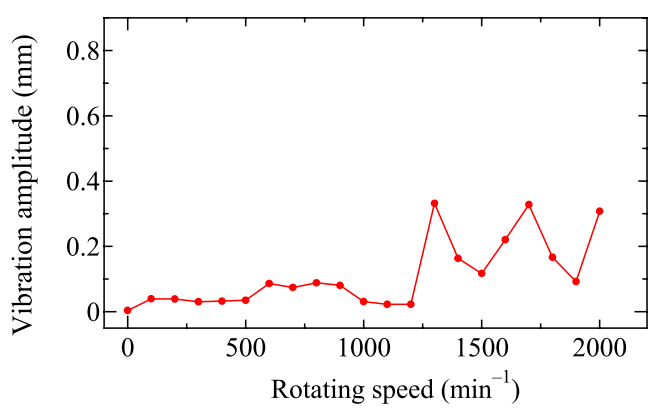

(a) Axial direction $z$

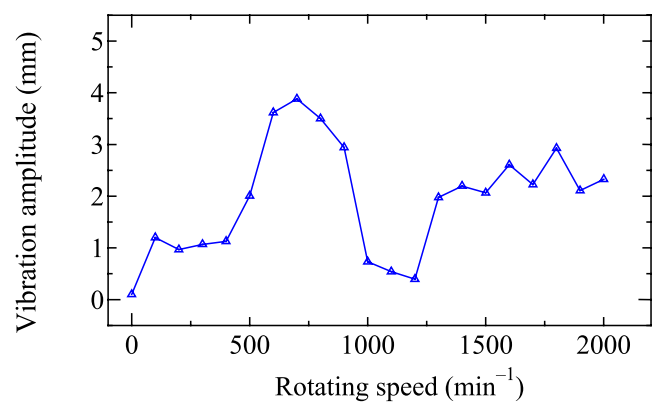

(b) Radial direction: $x$

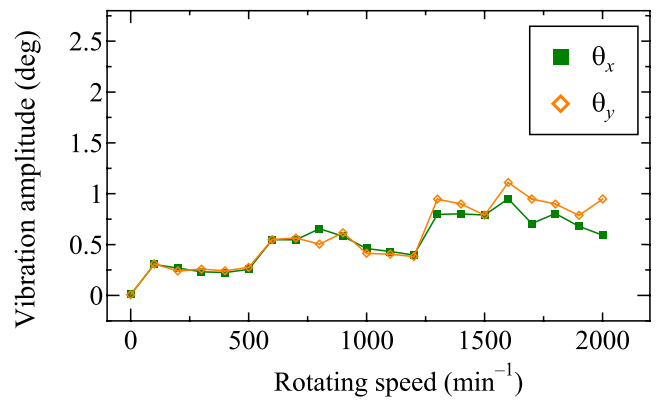

(c) Tilt: $\theta_{x}, \theta_{y}$

Fig. 11. Vibration amplitude

the increase in the vibration amplitude of the radial direction also affected the vibration amplitude of the axial direction slightly, the active control in the axial direction is capable of maintaining stable, levitated rotation. In the rotating speed range beyond $1300 \mathrm{~min}^{-1}$, however, levitation control of the axial direction became unstable. Thereby, the vibration amplitude of the radial direction also increased such that levitated rotation was broken at the speed of $2100 \mathrm{~min}^{-1}$. This is because the vibration amplitude of the axial direction became larger than the controllable range. The levitation control of the tilt directions was stable in all speed regions.

\section{Conclusion}

A permanent magnet hybrid type axial magnetically levitated motor was proposed. The FEM magnetic analysis showed that there was no significant interference between rotational torque, suspension force, and restoring torque, indicating that these four axes are therefore able to be controlled independently. The impulse response measured in the test rig showed quick response for the actively controlled degrees of freedom $\left(z\right.$ and $\left.\theta_{x}, \theta_{y}\right)$ and showed that the translation motion and the inclination motion can be controlled independently. Moreover, the time responses of each controlled axes were very quick. In addition, according to the result of levitated rotation characteristics, stable levitated rotation was observed 
in each actively controlled axes up to $2000 \mathrm{~min}^{-1}$.

Future works will be continued to obtain higher performance. The experimental setup will be also be modified for specific applications such as artificial heart and/or ultrapure water pump.

\section{References}

( 1 ) Y. Okada and K. Nonami: "Research Trends on Magnetic Bearings", JSME Int. Journal, Series C, Vol.46, No.2, pp.341-342 (2003)

( 2 ) A. Chiba, T. Fukao, et al.: "Magnetic Bearings and Bearingless Drives", Newnes (2005)

( 3 ) Y. Okada, T. Ohishi, and K. Dejima: "General Solution of Levitation Control of a Permanent Magnet(PM)-Type Rotating Motor", JSME Int. Journal Series C, Vol.38, No.3 (1995)

( 4 ) R. Schoeb and N. Barletta: "Principle and Application of a Bearingless Slice Motor", JSME Int. Journal Series C, Vol.40, No.4 (1997)

( 5 ) Y. Okada, S. Ueno, T. Ohishi, T. Yamane, and C.C. Tan: "Magnetically Levitated Motor for Rotary Blood Pumps", Artificial Organs, Vol.21, No.7, pp.739-745 (1997)

(6) S. Ueno and Y. Okada: "Characteristics and control of a bidirectional axial gap combined motor-bearing", IEEE/ASME Transactions on Mechatronics, Vol.5, No.3, pp.310-318 (2000)

( 7 ) K. Shimbo, I. Tomita, O. Ichikawa, C. Michioka, A. Chiba, and T. Fukao: "Axial Gap Length and the Maximum Torque of Shaftless Axial Gap Bearingless Motors", IEEJ Annual Meeting 1997, Paper No.1218 (1997) (in Japanes)

( 8 ) K. Chongkwanyuen, O. Ichikawa, C. Michioka, A. Chiba, and T. Fukao: "Inclination Control of Axial Gap Bearingless Motors", IEEJ Annual meeting 1997, Paper No.1219 (1997) (in Japanese)

( 9 ) M. Osa, T. Masuzawa, and E. Tatsumi: "Miniaturized Axial Gap Maglev Motor with Vector Control for Pediatric Artificial Heart", Journal of JSAEM, Vol.20, No.2, pp.397-403 (2012)

(10) H. Takada, N. Kurita, and T. Ishikawa: "Proposal of a Double tator Type Magnetically Levitated Axial Gap Motor", IEEJ Industry Applications Society Conference 2012, Paper No.Y-114 (2012) (in Japanese)

Nobuyuki Kurita (Member) received B.S. degree and M.S. degree

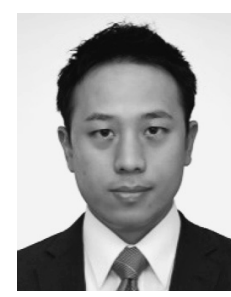
from Ibaraki University in 2001 and 2003, respectively. And he received his Ph.D. degree in engineering from Ibaraki University in 2006. He joined Gunma University as an assistant professor in 2009. His research interests include application of magnetic bearings and self-bearing motor. Dr. Nobuyuki Kurita is a member of IEEE and IEEJ.
Takeo Ishikawa (Senior Member) graduated from Tokyo Institute of

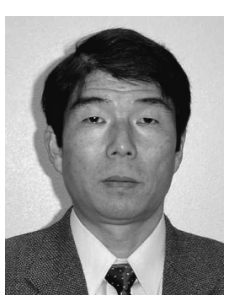
Technology in 1983. He joined to Gunma University in 1983, and now he is a professor. His research interest includes electrical machine and power electronics. He received the 1998 best paper award of IEEE Transaction on Vehicular Technology.

Hiromu Takada (Non-member) received B.S. from Gunma Univer-

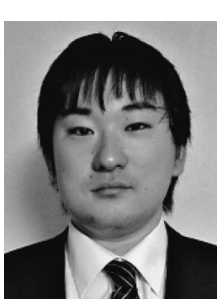
sity in 2010. He is Master student of graduate school of Gunma University. His research interests include application of magnetic bearings and self-bearing motor.

Genri Suzuki (Non-member) received B.S. from Gunma University

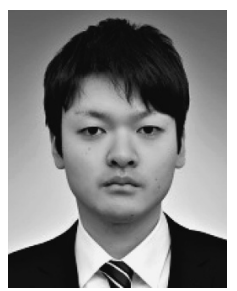
in 2012. He is Master student of graduate school of Gunma University. His research interests include application of magnetic bearings and self-bearing motor. 